\title{
Similarity Among Physical Phenomena Recognized on the Basis of the Classification of Existing Knowledge
}

\author{
Antonios Kanavouras $^{1}$ and Frank A. Coutelieris ${ }^{2 *}$ \\ ${ }^{1}$ Department of Food Science and Human Nutrition, Agricultural University of Athens, 55 IeraOdos Str., GR-11855, Athens, Greece \\ ${ }^{2}$ Department of Environmental Engineering, School of Engineering, University of Patras, 2 Seferi Str., GR-30100 Agrinio, Greece \\ *Corresponding author E-mail: fcoutelieris@upatras.gr
}

\author{
Article Info \\ Keywords: Classification matrix, Engi- \\ neering assets, Linear algebra, Physi- \\ cal phenomena, Similarity, \\ 2010 AMS: \\ Received: 20 February 2020 \\ Accepted: 31 August 2020 \\ Available online: 31 August 2020
}

\begin{abstract}
This work presents the synthetical mathematical analysis of available knowledge regarding physical phenomena of research interest. The work is not focusing on providing the phenomena according to the physical laws but rather because of them, hence, it is grounded on the philosophically defined concept of "similarity", and progresses to the mathematical treatments of those factors and parameters that are involved into the similarity validation among physical phenomena.A critical validation regarding the effectiveness of such an approach was also performed, in order to conceptualize the relevance of the factors and parameters interactions as a potential control tool against engineering-based hypothesis. Such factors and parameters are generated through the description and delimitation of the system of interest. A "matrix" is used for the classification of the existing knowledge regarding this system. It is consisted of the categorical descriptors of the system in question and the levels of these descriptors.A mathematical analysis of this "matrix" supports that all the existent perceptions of a physical phenomenon constitute a four-dimensional vector space. Within this space, the concept of similarity allows for the definition on which of a specific non-linear mapping that might be applied to strictly classify the existing knowledge about the phenomenon in question.Similarity is used here to define the conditions and the constrains that this mapping must satisfy. In conclusion, the applicability of the suggested approach on an engineering approach regarding a physical problem, was also demonstrated in this study.
\end{abstract}

\section{Introduction}

Experimentation is providing the evidently ground base for criticism and rational discussion. The outcome of such a process provides a reason-based scientific knowledge of any world of phenomena. Experiments are the fields of testing theories for their validity, need for changes or corrections. While a new theory may often derive along with a new phenomenon that needs explanation [1]. So far, scientific studies are based on either empirical or transcendental research approaches, aiming towards picturing the identity and/or the differences in time occurring throughout the phenomenon under investigation. Accordingly, research outcome is logically analyzed, and results are combined with empiricism and are all placed against the validity of the scientific hypothesis and its theoretical and practical interpretations. However, any reported result throughout the study is applied instantly and therefore records a series of incidents at certain time intervals. The empirical observations and/or their correlations are only to be further enhanced with an exceeding accumulation of data. In this context, incommensurability is a common situation impacting the objective view of researchers and the meaning they provide and the criteria they implement. The so called, social behavior of the researchers reflected on their commitments as well as on their objectivity is rather rich and diverse as may be realized via the multiplicity of research objects, fields and research subdivisions $[2,3]$. 
Furthermore, the attempt to induct the empirical justification into a theoretical interpretation is additionally present. Although vastly applied, statistical analysis results may contradict to the potential meaning and significance of the independent phenomenological events and individual steps or causes. It is more than obvious that the outcome of such applicational treatments is focusing on the "general" evolutionary trend, letting away of any valuable incorporation of any data differences that may be eventually lost when the general curve "trends" or the statistical significance level of the differences are presented. Supplementarytreatments on the curves with geometrical means in order to get additional estimations, such as kinetic parameters and phenomenological rates, are also applied. Therefore, derived conclusions may be highly biased by the selected treatments [4].

It is important to note that any physically executed experiment is actually a reproduction of the physical world under controlled conditions, hence within an artificial environment, namely the lab or an experimental field. What allows for such a transfer is actually, the acceptance that individual phenomena or their classes are referenced on the ground of similarities. Such similarities are constructed or perceived by the lead researcher on the ground of his/her fundamental considerations [5]. Such a reproduction is valid and accepted when it is applied in a standard and repeatable manner [6]. Having said that, the perception of the phenomena under study, is initiated with the essential initial hypothesis and is further build using the empirical observations that eventually lead to a logical understanding fitted well into a theory and practice [3].

Therefore, what is reality may well be perceived via an infinite number of phenomena, reproduced by the researchers in order to approach an objective view of the world, yet with questionable precision level. Moreover, and after having defined that two systems are similar, we may safely interchangeably infer the values of quantities in each of the physical systems from known values of each other. In other words, among two similar systems, it is allowed to draw conclusive outcomes for quantities based on a certain similarity mapping [7].

\section{Similarity concept}

Any experiment aiming in the collection of data, hence, empirical evidences, aims in identifying that information that may allow for further and broader view of the events well fitting into a theoretical method. The extrapolation of the knowledge gained in such a way, is valid and applicable, on the ground of similarity among the applied phenomena and fields and information may flow interchangeable among them

Nevertheless, the definite similarity of events and/or their classes background remains a question [8]. The answer apparently, may derive when considering that it is allowed to build comparative relationships and draw comparisons on the ground of the objects themselves or the hypothesis under which these objects are investigated [9].

As in any search attempt, the selection of criteria are fundamental as well for identifyingsimilarity. [7] suggested that a classification of the existing knowledge is necessary. Evidently, any inappropriate application of similarity criteria might easily lead to drawing of results lacking solid logical and/or meaningful ground. The role of any researcher is rather apparent in that process in order to avoid research waist and dead ends. Following that, any weak or erroneous used or selected similarity criteria shall guide the researchers into spending money and effort for to gain already existing knowledge, leading to resources' waste.

A number of knowledge classification attempts have been proposed, based on empirical and arbitrary rules and definitions [4]. In order to fulfil the requirement of similarity criteria selection and application, the work in hand suggests a solid mathematical treatment of identifying similarity and establishing robust similarity criteria through the application of fundamental Linear Algebra concepts (namely, vector spaces and mapping between them) on the relative philosophical aspects already arisen.

\section{Knowledge classification}

Scientists have aimed in studying any phenomenon in the Newtonian 3-dimensional world with certitude and clearness, completely in the light of principles, and, after having discovered the cause of the doubts and contradictions into which reason fell, to initiate an attempt to have them solved to its highest satisfaction possible.we will call "judgement" this inner logic that approaches the hypothesis with the thought that a thing is known to have a certain quality or attribute.

Judgement would be herein appointed as either "analytical", which when applied will expose the opposite sense to a logical contradictions, or "synthetical", in a sense that are additionally supporting that hypothesis, via extra treatments, such as in the case of mathematical laws applied as a judgemental tool. Furthermore, we shall call "proposals" the valid ways for approaching this logic. The a-posteriori proposals are those providing the possibility, while the a-priori proposals are those being based on inevitability and universality, distinguishing them as non - revisable. Overall, each and every way of approaching the logic may be either analytical or synthetic

Consistently, by combining the above, the following common general proposals derive: a) verification or the a-posteriori-analytical proposals, b) validation, or a-posteriori-synthetical proposals and c) prediction, or a-priori-analytical proposals. The current work is not focusing on providing the phenomena according to the physical laws but rather because of them, implying that our goal is a knowledge that is simultaneously non-empirical and a priori.

There may be no question regarding the phenomena non-existence before their expression and consequent empirical experience recorded and reported, only constructed through a local and cognitional process. Nevertheless, the level of distinction among the outcomes of the aforementioned process for various researchers, is not easily identified. Furthermore, a general principle of all the analogies rests on the synthetical unity of all phenomena according to their relation in time [10]. In order to overcome such obstacles, the use of mathematical synthetical treatments may allow for a synthetical representation of the perception of a phenomenon, [11].

In order to apply the above, we shall primarily need to define the functionality of the system under investigation and attempt to impend the empirical connections, sense and significance into its hypothesis. A summary of the organizational scheme for this work, is presented in Table 1, were the sequential (left to right and top to bottom) proposed developments, named the "epistemic structure" of the final engineering judgement, are acknowledged.

In specific, the developmental roadmap proposed, is divided in three (4.2) Stages. Within each development Stage and at the transitional 
Table 1: The developmental roadmap for organizing the work

\begin{tabular}{|l|l|l|l|l|l|}
\hline Stages & 1 & Proposals & 2 & Proposals & 3 \\
\hline Principles & & Physical & Intellectual & Mathematical & Engineering \\
\hline Proctorship & & Empirical & Analytical & Formulation & Relativistic \\
\hline $\begin{array}{l}\text { Functions of } \\
\text { Judgment }\end{array}$ & Potential & Synthetical & Necessity & $\begin{array}{l}\text { Hypothesis } \\
\text { potential } \\
\text { conditions }\end{array}$ \\
\hline Deliverables & System & & Categories & & Model \\
\hline
\end{tabular}

steps in-between, we shall aim in applying a judgemental process on the critical derived milestones ("Deliverables"). In order to do so we shall implement the essential scientific values for this particular system ("Principles") and the potential ways and methodology that will allow us to identify the interconnections ("Proctorship") among the systemic parts. This whole procedure will eventually define the outmost interesting deliverable, that may be used as an engineering tool and asset, the "Model" in Stage 3.

A detailed description of the fundamental activities we acknowledge as per Stage of Table 1, is following. That includes: i) the recognition and classification of the inherent systemic "ways-of-order" apprehendedas the categorical descriptors of the particular system and ii) the mathematical expressions that are evidently incorporating the principles and the logic and consequently the proof as a straight forward powerful application tool that is capable of assessing the system against the initial hypothesis.

\section{a) Stage 1}

We shall initially proceed with a validation first action focusing on the physical description of the system. Taken that experience is always proceeding the developed and emerged knowledge, the procedural progress along stages 1 and 2 needs to take int consideration the physical consider both the physical philosophies of the system along with empiricism's practices. This is only sto be solidly founded on both an appropriate experimental design and incorporate the much-needed monitoring means of the system's development in time and in space. That will be further discussed later in this work (see Section 5).

As mentioned above, the application of certain methodological, technical and mechanical borders during experimentation practice, place the upcoming expression of the system under the control of the selected border conditions. And therefore, the experimental outcome. Having worked in that manner, allows for a highly trustworthy data collected from the potential combinations within an experimental design hence, allowing us to collect and structure a holistic approach of the system. Through that overall picture, we may be certain of the particular and unique cohesive points of the phenomena occurring in such a well-defined system. From a certain point of view, it is the rationality of any model that may assist an "economic" experimentation.

\section{b) Stage 2}

The Deliverable in Stage 2 is the "Categories"; that are deriving from the research hypothesis itself. The relevant classification scheme shall then necessary and successfully derive the need and aims which when capably applied shall properly complete the description of the system. The description may be perceived as the reflection of the system on humans and that is why it may well be seen asa formal act of human cognition.

Nonetheless, the overall Categories need to contain the hypothesis' context possibilities for the system in question and regulate the system boundaries (classification frame), via impacting on each and every systemic participant, in-principal. Furthermore, the classification will reflect the theoretical solidness of the upcoming mathematical concept, independent of the empirical experience, in which case the deliverable model of Stage 3, ought to be capable of assessing the shaped categorical imperative of the hypothesis.

For an essential conceptual description of a system under investigation, we may convert the typical "in-process-out" context into a "system", via the incorporation of the finest, yet minimal, but still straightforward, systemic participants of generalized activities. Such activities are adequately described by the following four principal "categorical descriptors" in an equation form:

$$
\text { matter + energy } \underline{\text { relationships }}>\text { outcome }
$$

In order to advance the above core expression, the "categorical descriptors" have to be further developed with each and every thing in experience, the properties, the qualities, and the characteristics of any possibility in general.

Accordingly, when the aforementioned four categorical descriptors placed in a classification matrix of three, empirically defined, levels were basically adopted and later on drafted for the hypothesis in particular. Notably, the three levels also firmly satisfy the mathematical principle for the least linearly independent points to follow a non-linear relationship, as presented below.

Looking at each of the descriptors level, we may identify that a set of conditions exist. Moreover, our critical judgement's degrees of freedom are also present linking the descriptors to the hypothesis in question. Simply stated, all the three levels of each categorical descriptor, condense the impact for this systemic participant against the disclaiming of the hypothesis. Specifically, in Table 2, the four specific categories and their corresponding general levels are presented.

In that sense, the therefore formed 12 classes of the knowledge classification matrix there are the cohesions within the system context. These cohesions may be then expressed and presented with the appropriate mathematical expressions. Last but not least, it is an inter subjective 
Table 2: The knowledge classification matrix

\begin{tabular}{|l|l|l|l|}
\hline CATEGORIES & LEVELS & \multicolumn{2}{|l|}{} \\
\hline Matter (Quantity) & One & many & all \\
\hline Energy (Quality) & Reality & disallowance & restrictions \\
\hline Inherent potential (Relationships) & inter-dependent & reasons & intra-dependent \\
\hline Outcome (Processes) & Potential & existence & necessity \\
\hline
\end{tabular}

validation of the hypothesis, that is deriving through the cross sections of the classification cells , since the common ground among all classes' interactions may eventually deliver the basic and only physically meaningful and accountable potential conditions of knowledge.

The compulsory - exact analogy - filling-in of the specific hypothesis classes, demarcates the necessitating empirical and theoretical possibility conditions for the phenomena occur. As indicated in Table 1, the physical principles of the system along with the empirical (experimental and knowledge) available proctorship, shall be applied in this transitional step. The conceptual description of the system will, hence, border-lined upon a validation judgement assessment, corresponding to the classification scheme represented by Table 2, filled accordingly to the system, the hypothesis and the phenomena considered.

The validation analysis along with the categorical descriptions,allows the user to structure a system's awareness and consequently may generate the inherent mathematical relativistic expression that exist in the system and are based on their interrelationships. Having an and adequate consideration and performing a proper investigation of all classes, the process guarantees that, in principal, each and every systemic participant will accurately influence the boundaries of the model.

Finally, looking for a theoretical sound base of the system, the Categories may be used to provide the appropriate model, far beyond and above the empirical experience the study of the system may have or will allow. In that way, it is the model that shall assess the shaped categorical imperative of the hypothesis. This classification of the systemic properties will be the contextual guideline in logically conveying the physics in the system, from their mathematical description, to the engineering tool of Stage 3.

\section{c) Stage 3}

This stage is actually the use of the above two stages for a specific engineering problem. Therefore, a fulfilled $4 \times 3$ matrix can be produced, which contains all the necessary information about the knowledge regarding a specific physical phenomenon.

\section{The mathematics of classification}

Given the goals of this work, let us initially consider a physical and/or chemical phenomenon. What we have actually in mind is the perception of an incident or a series of interconnected incidents that belong to a specific class of physico chemical phenomena. This perception could be described by Equation (3.1) when and only when, the exclusive phenomenon's characteristics may be incorporated within the categorical descriptors specified for this phenomenon. Accordingly, this means that one has to quantify the "matter" and the "energy" involved, to identify a macroscopic magnitude that serves as the "outcome" of the phenomenon as well as to express a valid "relationship" among the parameters involved in the expression of the phenomenon in question. There should be a linear independence among these descriptors because (a)matter is a manifest object, (b) energy is a manifest object, as well, (c) relationship is a mathematical object being valid independently regarding where it is applied for, and (d) outcome is defined subjectively, yet, in-line with the scientific hypothesis, under which the phenomenon is studied.

To the extent that there may exist an infinite number of perceptions of any phenomenon, there are also infinite tetrads, consisted of values for the matter, energy, relationships and outcome categorical descriptors. Each one of these tetrads forms a vector of the form $\underline{u}=\{m, e, R, o\}$, where a one-by-one correspondence exists between the values of vector components and a specific perception. Then, we may now determine a set $V$, consisted of all these vectors $\underline{u}$. There is a non-obvious relation between vectors $\underline{u}$ and time: each vector corresponds to the whole amount of knowledge regarding the phenomenon, which is available at a specific time. This means that every element of $V$ reflects a precise value of time. In that sense, vectors $\underline{u} \in V$ can be considered as functions of time.

Hence, it could now become able to underline that the set $V$ is an ordered set where an arrangement, denoted by $\prec$, can be defined as follows:

$$
\text { for } \hat{t}_{1}<\hat{t}_{2} \Leftrightarrow \underline{u}_{1}\left(\hat{t}_{1}\right) \prec \underline{u}_{2}\left(\hat{t}_{2}\right)
$$

Note also that each $\underline{u} \in V$ embeds all the vectors of lower values, i.e. all the knowledge previously obtained and currently available. By considering a physical phenomenon along with a disclaiming hypothesis superimposed (as previously stated), every new perception consists of the current knowledge about the phenomenon plus a new contribution

$$
\underline{u}_{i}\left(\hat{t}_{i}\right)=\underline{u}_{i-1}\left(\hat{t}_{i-1}\right)+A
$$

where $\underline{u}_{i}\left(\hat{t}_{i}\right)$ is the current of knowledge, $\underline{u}_{i-1}\left(\hat{t}_{i-1}\right)$ is the previously obtained of knowledge and $A$ is the amount of knowledge added to the previous knowledge in order to produce the current one.

Having described the arrangement of the set $V$, we can define an internal operation $\oplus$ as follows

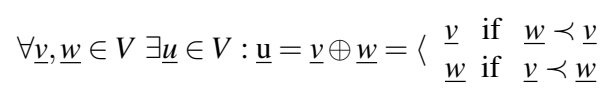


This operation describes mathematically the knowledge evolution through time, which is obtained by "adding" new contribution on the existing accumulated knowledge.

Regarding its properties, this operation

(a) is commutative

$$
\underline{v}, \underline{w} \in V \underline{v} \oplus \underline{w}=\underline{w} \oplus \underline{v}
$$

Proof:

If

$$
\left.\underline{w} \prec \underline{u} \Longrightarrow \begin{array}{l}
\underline{u} \oplus \underline{w}=\underline{u} \\
\underline{w} \oplus \underline{u}=\underline{u}
\end{array}\right\} \Rightarrow \underline{u} \oplus \underline{w}=\underline{w} \oplus \underline{u}
$$

If

$$
\left.\underline{u} \prec \underline{w} \Longrightarrow \begin{array}{l}
\underline{u} \oplus \underline{w}=w \\
\underline{w} \oplus \underline{u}=\underline{w}
\end{array}\right\} \Rightarrow \underline{u} \oplus \underline{w}=\underline{w} \oplus \underline{u}
$$

(b) is associative

$$
\forall \underline{v}, \underline{w}, \underline{u} \in V \underline{v} \oplus(\underline{w} \oplus \underline{u})=(\underline{w} \oplus \underline{v}) \oplus \underline{u}
$$

Proof:

If

$$
\left.\underline{v} \prec \underline{w} \prec \underline{u} \Longrightarrow \begin{array}{l}
\underline{v} \oplus(\underline{u} \oplus \underline{w})=\underline{v} \oplus \underline{u}=\underline{u} \\
(\underline{w} \oplus \underline{v}) \oplus \underline{u}=\underline{w} \oplus \underline{u}=\underline{u}
\end{array}\right\} \Rightarrow \underline{v} \oplus(\underline{u} \oplus \underline{w})=(\underline{w} \oplus \underline{v}) \oplus \underline{u}
$$

The other cases for the arrangement $(\underline{v} \prec \underline{w} \prec \underline{u}, \underline{v} \prec \underline{u} \prec \underline{w}, \underline{u} \prec \underline{v} \prec \underline{w}, \underline{w} \prec \underline{v} \prec \underline{u}, \underline{u} \prec \underline{w} \prec \underline{v}$ and $\underline{w} \prec \underline{u} \prec \underline{v}$ ) can be proven in the same way. (c) has identity element

$$
\forall \underline{v} \in V \exists \underline{0} \in V: \underline{v} \oplus \underline{0}=\underline{0} \oplus \underline{v}=\underline{v}
$$

Proof:

The element $\underline{0}$ stands for the trivial knowledge that tends to zero, thus not actually favoring knowledge evolution.

(d) each vector in $V$ has inverse elements

$$
\forall \underline{v} \in V \exists(-\underline{v}) \in V: \underline{v} \oplus(-\underline{v})=(-\underline{v}) \oplus \underline{v}=\underline{0}
$$

Proof:

Vector $(-\underline{v})$ in fact represents a singularity on the knowledge evolution, i.e. one point the knowledge of which tends to collapse all the existing knowledge.

As far as $\underline{u}$ is a vector, its regular norm $\lambda=\|\underline{u}\| \in \mathbb{R}$ is the amount of knowledge that is embedded in the perception of the phenomenon. This norm allows for the quantification of the amount of knowledge between any two elements of $V$, and given that $V$ is an ordered set, the ratio of such quantities is a realization of knowledge evolution. In terms of mathematics, the above can be expressed as

$$
\text { if } \underline{u} \prec \underline{v} \text { with } \underline{u}, \underline{v} \in V \text {, then } \exists \mu \in(1,+\infty): \mu=\frac{\|\underline{u}\|}{\|\underline{v}\|}
$$

We can now define another operation $\otimes$ as follows:

$$
\forall \underline{v}, \underline{w} \in V \exists \mu \in R: \underline{w}=\mu \bigotimes \underline{v} \Leftrightarrow \mu=\frac{\|\underline{w}\|}{\|\underline{v}\|}
$$

which describes the relative importance of the difference in knowledge amounts between two particular time periods, i.e. between two perceptions expressed in different times.

Having determined the set $V$ as well the operations $\bigoplus$ by eq. (4.3) and $\otimes$ by eq. (4.12), we can now that the group $\{V, \oplus, \otimes\}$ is a $4^{\text {th }}$ dimensional vector space. It is assured because, on top of the properties of operation $\bigoplus$ presented through eqs. (4.4) $-(4.10)$, it is straightforward to prove that operation $\otimes$ satisfies the following relations 


$$
\begin{gathered}
\lambda(\mu \bigotimes \underline{v})=\lambda \mu \bigotimes \underline{v} \\
(\lambda+\mu) \bigotimes \underline{v}=\lambda \bigotimes \underline{v} \bigoplus \mu \bigotimes \underline{v} \\
\lambda(\underline{u} \bigoplus \underline{v})=\lambda \underline{u} \bigoplus \mu \underline{v}
\end{gathered}
$$

Note also that the dimensions of this vector space are defined by the number of categorical descriptors, as visualized in eq. (3.1). One basis of this space consists of the four vectors $\underline{e}_{m}=\{m, 0,0,0\}, \underline{e}_{e}=\{0, e, 0,0\}, \underline{e}_{R}=\{0,0, R, 0\}$ and $\underline{e}_{o}=\{0,0,0, o\}$. We may then report that,

(a) the components of the basis are linearly independent since they reflect the independent categorical descriptors, as previously stated

(b) any vector in the space is described by a unique linear combination of the basis vectors.

Finally, it could be able to identify a non-linear mapping $m_{p}^{I n}$ over the space $\{V, \oplus, \otimes\}$, as follows

$$
\mathbb{R} x V \stackrel{m_{p}^{I n}}{\longrightarrow} M_{3 x 1}(V): m_{p}^{I n}(\underline{v})=\left\{\begin{array}{lll}
\lambda_{1} \underline{v}, & \lambda_{2} \underline{v}, & \lambda_{3} \underline{\underline{v}}
\end{array}\right\}
$$

with

$$
\begin{gathered}
\lambda_{1} \rightarrow 0 \\
\forall \lambda_{2} \in \mathbb{R} \exists M>0: \lambda_{2}>M \\
\lambda_{3} \rightarrow+\infty
\end{gathered}
$$

The first component of the product, given by eq. (4.16) under the constraint (4.17), corresponds to a very low amount of knowledge that tends to zero but it should always be non-zero, meaning that it is even at its minimum level enough to initiate the scientific/research interest for studying the specific phenomenon. The next component describes the currently available knowledge while the last one, along with constraint (4.19) represents the nearly infinite amount of knowledge, needed to be obtained in order to finalize the research on this topic. The above eqs. (4.17)-(4.19) assure the compatibility of "one-many-all", commonly used in modern philosophy as a well-known Kantian wit [12].

The above mapping [eq. (4.16)] produces a matrix with four lines, each one standing for each of the elements $\{m, e, R, o\}$, and three columns, the first for the vector $\lambda_{1} \underline{v}$, the second for the $\lambda_{2} \underline{v}$ and the third for the $\lambda_{3} \underline{v}$. As previously stated, the first column refers to a vector containing the minimum non-zero knowledge of the phenomenon, where only one variable, along with only one mathematical equation produced by one simple conservation law or a relative mass/energy balance, are considered adequate to describe the particular perception. In fact, the first column of the matrix produces a rather primitive ideal outcome, which can roughly represent the phenomenon. The second column refers to the maximum finite knowledge currently available, where a finite number of variables are selected to describe the phenomenon and, therefore, a system of equations is produced, while a single one parameter is again selected to macroscopically describe the phenomenon. Briefly speaking, the second vector is a more accurate and more efficient representation of the phenomenon under consideration. Finally, the third column describes the absolutely holistic perception of the phenomenon, taking into account an infinite number of variables that define a system of equations with infinite dimension. In other words, the third vector describes the overall currently available knowledge about a phenomenon, identifying all the parameters' impact, although not necessarily known in full details.

Following the order of set $V$, it is

$$
\lambda_{1} \underline{v} \prec \lambda_{2} \underline{v} \prec \lambda_{3} \underline{v}
$$

where each vector contains the knowledge represented by the previous vectors.

We support that the non-linear mapping defined by eqs. (4.16), (4.17)-(4.19), quantifies the similarity between any two perceptions of the phenomenon in question, in terms of the way the evolution of knowledge occurs withing and cross the phenomenon's expressions.

It is also important that the definition of such a mapping is not unique. In order for the researcher to select the most suitable and appropriate mapping, i.e. to select values for $\lambda_{1}, \lambda_{2}, \lambda_{3}$ as well as $m, e, R$ and $o$, a complicated methodology must be followed, as described in detail elsewhere [13]. The final aim of the application of this methodology is to identify potential lacks of knowledge, in order to direct the relative research and to avoid resources waste. 
Table 3: Classification matrix for adsorption in granular media

\begin{tabular}{|c|c|c|c|}
\hline & $\lambda_{1} \underline{v}$ & $\lambda_{2} \underline{v}$ & $\lambda_{3} \underline{v}$ \\
\hline Matter & A & A, solid & A, solid, products $\mathrm{B}_{i}$ \\
\hline \multirow[t]{3}{*}{ Energy } & $\begin{array}{l}\text { Diffusion: } \\
\underline{j}_{A}=-D_{A} \nabla C_{A}\end{array}$ & $\begin{array}{l}\text { Diffusion: } \\
\underline{j}_{A}=-D_{A} \nabla C_{A}\end{array}$ & $\begin{array}{l}\text { Diffusion: } \\
\underline{j}_{i}=-D_{i} \nabla C_{i}\end{array}$ \\
\hline & $\begin{array}{l}\text { Convection: } \\
\underline{j}_{A}=U_{A} C_{A}\end{array}$ & $\begin{array}{l}\text { Convection: } \\
\underline{j}_{A}=U_{A} C_{A}\end{array}$ & $\begin{array}{l}\text { Convection: } \\
\underline{j}_{i}=U_{i} C_{i}\end{array}$ \\
\hline & $\begin{array}{l}\text { Instantaneous adsorp- } \\
\text { tion } \\
C_{A}(r=R)=0\end{array}$ & $\begin{array}{l}\text { Adsorption by isotherm } \\
D_{A} \underline{n} \cdot \nabla c_{A}=\frac{k}{K} c_{S} \\
\Theta_{e q}=\frac{K c_{b}}{1+K c_{b}}\end{array}$ & $\begin{array}{l}\text { Reaction of first order } \\
A \rightarrow B, \\
\text { with reaction rate } \\
R_{n}=k_{0} e^{\frac{-E_{A}}{R T}} c_{A}\end{array}$ \\
\hline $\begin{array}{l}\text { Relation } \\
\text { ships }\end{array}$ & $\begin{array}{l}\frac{d C_{A}}{d t}+\underline{U}_{A} \cdot \nabla C_{A}= \\
D_{A} \nabla^{2} C_{A}\end{array}$ & $\begin{array}{l}\frac{d C_{A}}{d t}+\underline{U}_{A} \cdot \nabla C_{A}= \\
D_{A} \nabla^{2} C_{A}\end{array}$ & $\begin{array}{l}\frac{d C_{i}}{d t}+\underline{U}_{i} \cdot \nabla C_{i}= \\
D_{i} \nabla^{2} C_{i}\end{array}$ \\
\hline Outcome & $C_{A}(\underline{r}, t)$ & $S h_{o}=\frac{k_{o} \cdot L}{D}$ & 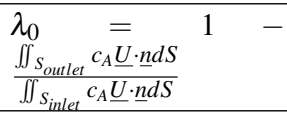 \\
\hline
\end{tabular}

\section{Application \& discussion}

In order to further support our approach, we shall now provide an application of the above described methodology to the physico-chemical phenomenon of mass transport in porous materials. The geometry where the phenomena occur is described by a swarm of uniform solid spheres. It is assumed that a Newtonian dilution flowsunder laminar flow conditionsthroughout the void space. There is a substance A diluted in the fluid phase, that flows in the pore volume and can be adsorbed on the solid surface of the grains. In general, mass transport of A is driven by diffusion and convection in the fluid phase, and by the considered sorption mechanism on the fluid-solid interfaces. All the above can be mathematically summarized in a generalized form of a vector $\underline{u} \in V$ described as [14]:

$$
\begin{array}{r}
\underline{u}=\left\{A, \text { mass transport mechanisms, } \frac{d C_{A}}{d t}+\underline{U}_{A} \cdot \nabla C_{A}=D_{A} \nabla^{2} C_{A} \pm R_{A}\right. \\
\text { with appropriate BCs, macroscopic quantity described adsorption }\}
\end{array}
$$

In this context,the output of the non-linear mapping is presented in the following $4 \mathrm{X} 3$ matrix:

The highly significant point to underline for Table 3, is that it unfolds through levels. In terms of mathematics given in eq. (4.16), the mapping can be defined through the identification of vector $\underline{v}$ and real numbers $\lambda_{1}, \lambda_{2}$ and $\lambda_{3}$. Precisely,

$$
\underline{v}=\left\{A, \text { convective-diffusion with instantaneous adsorption, } \frac{d C_{A}}{d t}+\underline{U}_{A} \cdot \nabla C_{A}=D_{A} \nabla^{2} C_{A} \text { with } C_{A}(r=R)=0, C_{A}(\underline{r}, t)\right\}
$$

therefore

$$
\begin{gathered}
\lambda_{1}=1 \\
\lambda_{2}=\text { any finite number } \\
\lambda_{3} \rightarrow \infty .
\end{gathered}
$$

\section{Conclusions}

Systemic characteristics, predicates, attributes, qualities, or properties control the progress of the phenomena. A justified selection of them has to be included at certain fine-tuned levels, during the experimental phase, for an ably economic combination at the experimental phase and a comparable outcome data. Such a holistic approach shall adequately reveal the cohesiveness among the phenomena, rather than their simple description. Common ground establishments, under which certain common characteristic are shared among classes, may somehow be related to one another. Creating and applying precise conditions,that shall define the progress of any experimental phase, have a well-defined and significant role in allowing the system in question to unroll and progress on the base of its systemic properties, towards the particular processes' outcome, at empirically controlled environments.

Finally, the matrix may possess an additional added value, which is to confirm the experimental set-up that shall lead researchers to set the out most pragmatic input to an engineering model. The engineering tool's usefulness relies on the fact that it may work far beyond the judgemental capabilities of typical predictions and verifications that a mathematical model typically provides, yet such a model allows for 
the identification and management of the experimentation risk options. These should accordingly hold the overall optimum engineering that shall provide the minimum possibility of disclaiming the fundamental disclaiming hypothesis.

Utilizing the herein presented concept of similarity between physical phenomena, when interested in engineering approaches, this work also presents a strict mathematical formulation for the classification of the existing knowledge regarding a physical phenomenon.

This work proves that all the potential perceptions of a phenomenon along with specific operations (analogous to summation and multiplication) with proven properties, constitute a vector space. By taking into account that this space is of four dimensions, a non-linear mapping over this vector space has been also defined to describe mathematically the similarity concept. The value of such a mathematical treatment relies on its capability to provide a deep insight on a specific phenomenon, while at the same time assists the researchers to set up an experimentation plan focusing on the knowledge gaps and work towards filling in these gaps properly. Finally, the proposed model is therefore an engineering tool for avoiding repetition of results and managing waste of research effort, in general.

\section{References}

[1] R. Giere, Scientific Perspectivism, University of Chicago Press, Chicago, USA, 2006.

[2] H. Douglas, The irreducible complexity of objectivity, Synthese, 138 (2004), 453-473.

[3] T. S. Kuhn, The Structure of Scientific Revolutions, University of Chicago Press, Chicago, USA, 1962.

[4] P. Feyerabend, Explanation, Reduction and Empiricism, Scientific Explanation, Space, and Time, (Minnesota Studies in the Philosophy of Science, Volume III), H. Feigl, G. Maxwell (editors), University of Minneapolis Press, USA, 1962.

[5] P. Kroes, Structural analogies between physical systems, Brit. J. Philos. Sci., 40 (1989), 145-154.

[6] I. Hacking, Representing and Intervening. Introductory Topics in the Philosophy of Natural Science, Cambridge University Press, UK, 1983.

[7] S.G. Sterrett, Models of machines and models of phenomena, Stud. Philos. Sci., 20 (2006), 69-80.

[8] C. Glymour, On some patterns of reduction, Philos. Sci., 37 (1970), 340-353.

[9] S.G. Sterrett, Physical models and fundamental laws: Using one piece of the world to tell about another, Mind Soc., 3 (2002), $51-66$.

[10] I. Kant, The Critique of Pure Reason (Translated by J. M. D. Meiklejoh), University of Adelaide Press, Adelaide, AUS, 2014.

[11] A.S. Troelstra, H. Schwichtenberg, Basic Proof Theory, Cambridge University Press, Cambridge, UK, 2000.

[12] K.R. Popper, Conjectures and Refutations: The Growth of Scientific Knowledge, Harper, New York, USA, 1963.

[13] A. Kanavouras, F.A. Coutelieris, Systematic transition from description to a prediction engineering model for the oxidation in packed edible oils, J. Food Chem., 229 (2017), 820-827.

[14] R.B. Bird, W.E. Stewart, E.N. Lightfoot, Transport Phenomena, Wiley, New York, USA, 1960. 